\title{
SOME NOMENCLATURAL CORRECTIONS TO THE CARIBBEAN FLORA
}

\author{
A. L. BORHIDI \\ Department of Plant Biology, Institute of Biology, Faculty of Sciences \\ University of Pécs, Pécs, Hungary; E-mail: borhidi@gamma.ttk.pte.hu
}

(Received 2 August, 2015; Accepted 31 August, 2015)

The validation of two Bouvardia (Rubiaceae) species of Mexico is carried out recommended by Govaerts because the placing of their type materials was not included into the protologues. New names are proposed for a Mexican Bouvardia and a neotropical Uragoga (both Rubiaceae) species having illegitimate names. A correction of author's name is proposed for a Miconia (Melastomataceae) species of Cuba.

Key words: additions, Bouvardia, corrections, Miconia, nomenclature, Uragoga

\section{VALIDATION OF THE FOLLOWING INVALID NAMES OF THE MEXICAN FLORA}

Bouvardia diversiflora Borhidi et Lozada-Pérez, Acta Bot. Hung. 53: 44 (2011). - Type: México. Guerrero, Mpio. Atoyac, 16 km al NE de El Paraiso. Alt. 1100 m snm. Col.: J. C. Soto Nuñez (10142), con S. Román G., 19. Aug. 1985. Holotype: MEXU, isotype: FCME.

Bouvardia fragilis Borhidi et S. Salas-Morales, Acta Bot. Hung. 53: 45 (2011). - Type: México. Oaxaca, Distr. Pochutla, Mpio. San Miguel del Puerto, Cerro Lobo, Bosque mesófilo de montaña, suelo negro. Coord.: $16^{\circ} 01^{\prime} 16.4^{\prime \prime}$ N, 96 06' 33.1" W, alt.: 2092 m snm. Col.: José Pascual (779), 27 May 2003. Holotype: MEXU, isotype: SERO.

\section{NEW NAMES PROPOSED FOR THE FOLLOWING TWO SPECIES OF NEOTROPICAL RUBIACEAE}

Bouvardia perelegans Borhidi, nomen novum, hoc loco. - Replaced name: Bouvardia elegans Borhidi, Acta Bot. Hung. 53: 52 (2011), nomen illeg. 
non Bouvardia elegans Hendr. et Andr. Hendr., Gard. Chron. 1870: 446 (1870). - Type: México. Oaxaca, Mpio. San Jerónimo Coatlán, Diatr. Miahuatlán, 24 $\mathrm{km}$ al SO de San Jerónimo Coattlán, brecha a Piedra Larga, Cañada de bosque mesófilo en bosque Pino-encino, suelo amarillo arcilloso. Alt.: $1600 \mathrm{~m} \mathrm{snm}$. Col.: Alvaro Campos V. (4628), 2 June 1992. Holotype: MEXU.

Palicourea winkleri Borhidi, nomen novum, hoc loco. - Replaced name: Palicourea buchtieni (H. Winkler) Borhidi, Acta Bot. Hung. 54: 83 (2012), nom. illeg. non Palicourea buchtieni Standley, Publ. Field Mus. Nat. Hist. Bot. ser. 7: 317 (1931). - Basionym: Uragoga buchtienii H. Winkler, Repert. Spec. Gen. Nov. Regni Veget. 8: 4 (1910). - Type: Bolivia (La Paz), San Antonio bei Mapiri, 850 m. Col.: O. Buchtien (1608), Dec. 1907. Lectotype selected by Standley: B: destruido; isotypes: F, US n.v. - Syn: Psychotria buchtienii (H. Winkler) Standley, Publ. Field Mus. Nat. Hist. Bot. ser. 7: 303 (1931).

\section{CORRECTION OF AUTHOR'S NAME}

Majure L. C. et al. published recently an extremely important article about molecular phylogenetic relations between certain groups of Ossaea and Miconia species of the Greater Antilles (Majure et al. 2015). In conclusion they proposed the transfer of 20 species from Ossaea, Calycogonium and other genera to Miconia, creating new names and new combinations. These nomenclatural changes all are correct, except the following one on the page 622, where we find:

Miconia pseudopinetorum (Alain) Judd, Bécquer et Majure, comb. nov. - Basionym: Ossaea pseudopinetorum Borhidi et O. Muñiz, Acta Bot. Acad. Sci. Hung. 17: 20 (1972 (1971)). - Type: Cuba (Prov. Guantánamo), Moa, Cupeyal de Norte, Río Toa, fr. Col.: A. Borhidi et al. (SV-27096), 9 February 1970. Holotype: HAC!.

Ossaea pinetorum Alain, Contr. Ocas. Mus. Hist. Nat. Colegio "De La Salle" 15: 8 (1956). - Type: Cerca del Batey de "Corea", Sierra de Mícara, Mayarí, fr. Col.: Bro. Alain \& López Figuerias (4636), 27 December 1955. Holotype: HAC!, HAJB!; isotype: GH!. Non Miconia pinetorum Naudin, Ann. Sci. Nat., Bot. sér 3, 16: 229 (1851).

Obviously, the correct form of the new combination is:

Miconia pseudopinetorum (Borhidi et O. Muñiz) Judd, Bécquer et Majure, hoc loco.

Alain cannot be the author of the name, because he never published it. Other question is, that I am not convinced, whether Ossaea pinetorum Alain and Ossaea pseudopinetorum Borhidi et O. Muñiz would be conspecific. 


\section{REFERENCES}

Borhidi, A. (2011): Transfer of the Mexican species of Psychotria subgen. Heteropsychotria to Palicourea based on morphological and molecular evidences. - Acta Bot. Hung. 53: 241-250. http://dx.doi.org/10.1556/ABot.53.2011.3-4.4

Borhidi, A. (2012): Rubiáceas Mexicanas. Second, enlarged edition. - Akadémiai Kiadó, Budapest, 608 pp.

Borhidi, A., Lozada-Pérez, L. and Salas-Morales, S. (2011): Estudios sobre Rubiáceas Mexicanas XXXI. Trichosiphon y Gymnosiphon sect. nov. subgen. Bouvardiastrum (Bouvardia Salisb.) con seis especies nuevas. - Acta Bot. Hung. 53: 41-61. http://dx.doi. org/10.1556/ABot.53.2011.1-2.4

Govaerts, R. (2014): World checklist of Rubiaceae. - Royal Botanic Gardens, Kew. http://apps. kew.org/wcsp

Majure, L. C., Neubig, K. M., Skean, J. D. Jr., Bécquer, E. R. and Judd, W. S. (2015): Evolution of the Sandpaper clade (Miconieae, Melastomataceae). - Int. J. Plant Sci. 176(7): 607-626. http://dx.doi.org/10.1086/682148 


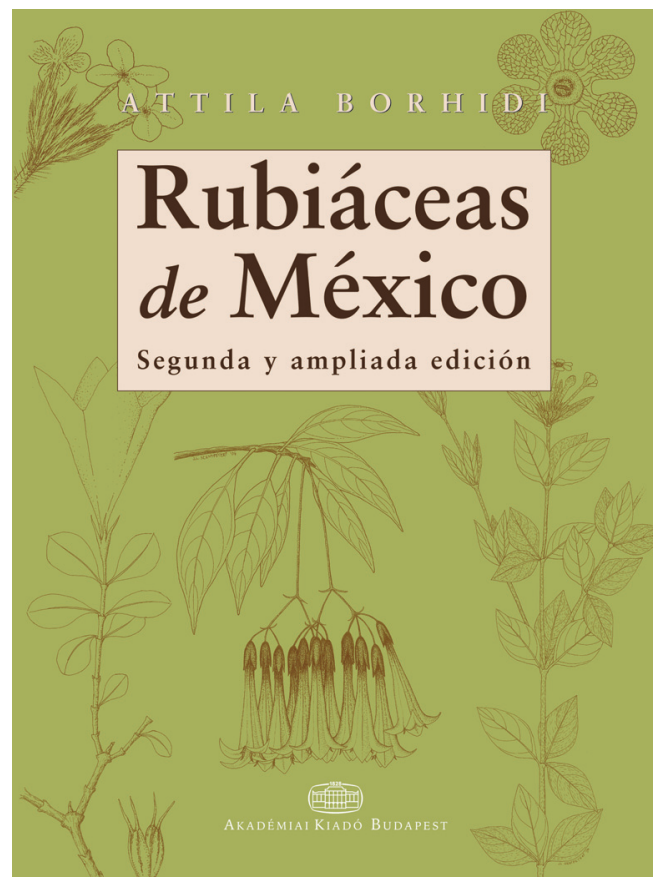

Este libro es la segunda y ampliada edición de la primera monografía completa de la familia Rubiaceae (con más de 13000 especies y 650 géneros en el mundo) la quinta más importante en la flora de los Estados Unidos de México. Durante el período de 5 años pasados desde la primera edición (2006), el número de los géneros tratados se aumentó de 103 a 113 y de las especies de 582 a 666, en consecuencia de la actividad exploradora de las distintas instituciones y sociedades naturalistas. La obra abarca también más de 2700 sinónimos y 127 ilustraciones artísticas y una reseña fitogeográfica sobre el papel e importancia de la família en la diversidad extraordinaria de la flora Mexicana en la flora y vegetación tropical de la área del Caribe. Esta edición tiene nuevas claves de determinación más prácticas, sigue los resultados de las investigaciones moleculares con gran sensibilidad, resolviendo también los problemas actuales taxonómicos discutidos, sobre los complejos de Deppea, Rondeletia Bouvardia y Psychotria.

El libro es un recurso indispensable de los conocimientos botánicos y taxonómicos para los expertos científicos y personales trabajando en los campos de la botánica, ecología, fitoquímica, agronomía y silvicultura, de la cartografía de los recursos ambientales, de la planificación física, etc. asimismo para los estudiantes universitarios y de la enseñanza superior. El libro es una obra coplementaria importane del tomo correspondiente de la Flora Mesoamericana.

Can be ordered directly from the publisher at www.akkrt.hu 